\title{
NÚMERO DE OVULACIONES POR CICLO ESTRUAL EN CUYES (Cavia porcellus) ANDINA Y PERÚ
}

\author{
Number of Ovulations per Estrous Cycle in Andina and Peru Guinea Pig \\ (Cavia porcellus) BREEDS
}

\author{
Eduardo Araníbar ${ }^{1}$, Luisa Echevarría C. ${ }^{1,2}$
}

\section{Resumen}

El estudio tuvo por objetivo determinar el número promedio de folículos que llegan a ovular en cuyes de las razas Andina y Perú, con el fin de saber si la diferencia en el tamaño de camada entre estas razas se debe, en parte, al número de ovulaciones. Se utilizaron 15 animales de 10 semanas de edad por raza. Se consideró como día 0 del ciclo estral el día de la ovulación, el cual se determinó mediante citología vaginal y ausencia de la membrana vaginal. En el día 8 del ciclo estral se realizó citología vaginal para comprobar que los animales estaban en diestro y se sacrificaron para obtener los ovarios. Estos fueron fijados con formol buferado, se hicieron cortes seriados y se colorearon con hematoxilina eosina. Las muestras histológicas se observaron en microscopio óptico para el conteo de los cuerpos lúteos. En la raza Andina se encontró $3.6 \pm 0.9$ cuerpos lúteos y en la Perú se encontró $2.8 \pm 0.9$ cuerpos lúteos $(\mathrm{p}<0.05)$. La diferencia en el tamaño de camada entre las dos razas se podría deber, en parte, a que la raza Andina tiene mayor número de ovulaciones por ciclo estral que la raza Perú.

Palabras clave: cuy, Perú, Andina, ovario, cuerpo lúteo

\section{Abstract}

The aim of the study was to determine the average number of ovulating follicles in the Andina and Peru guinea pigs breeds in order to know if the difference in litter size between the two breeds is partly due to the number of ovulations. Fifteen animals of 10 weeks of age per breed were used. The day of ovulation was considered as day 0 of the estrous cycle and was determined through vaginal cytology and absence of the vaginal membrane. In day 8 of the estrous cycle vaginal cytology was done to verify that the

\footnotetext{
${ }^{1}$ Facultad de Medicina Veterinaria y Zootecnia, Universidad Peruana Cayetano Heredia, Lima, Perú

${ }^{2}$ E-mail: luisa.echevarria@upch.pe
} 
female was in diestrus and then animals were slaughtered to obtain the ovaries. These were fixed with buffered formalin and histological sections of ovaries were stained with hematoxylin eosin. The samples were observed in optical microscope and corpus lutea (CL) were counted. The Andina breed had 3.6 \pm 0.9 CLand the Peru breed had 2.8 $\pm 0.9 \mathrm{CL}$ $(\mathrm{p}<0.05)$. The difference in litter size between the two breeds would be partially due to the higher number of ovulations per estrous cycle in the Andina breed as compared to the Peru breed.

Key words: guinea pig, Peru, Andina, ovary, corpus luteum

\section{INTRODUCCIÓN}

El cuy (Cavia porcellus) es un mamífero herbívoro originario de la zona andina, que se caracteriza por ser una especie precoz, prolífica y de ciclos reproductivos cortos. El Perú es el principal productor y consumidor de carne de cuy a nivel mundial, habiéndose estimado una producción de 15500 toneladas de carne en 2003 (INIA, 2013), con una población de 22 millones de cuyes (Chauca, 1997).

El ciclo estrual del cuy tiene una duración promedio de $16.1 \pm 0.2$ días con un rango de 13 a 22 días (Shi et al., 2000). La fase de proestro dura 1-1.5 días, estro 8-24 horas, metaestro 1-1.5 días y diestro 13-15 días (Künhnel y Mendoza, 1992). La membrana vaginal está presente en la periferie de los genitales de la hembra y se ausenta por 3 a 7 días (4.1 días en promedio) coincidiendo con la etapa de celo (Luna et al., 2003). La ovulación ocurre 1 a 1.5 días posteriores a la apertura vaginal (Sadeu et al., 2007). Esta especie tiene 3.14 ovulaciones en promedio por ciclo estrual, pudiendo llegar hasta 8 ovulaciones por ciclo. Asimismo, el promedio de crías por camada es de tres (Noonan, 1994).

La citología vaginal permite determinar las fases del ciclo estrual. En el cuy, el proestro se caracteriza por la presencia de abundante mucus y células intermedias grandes o pequeñas; en el estro se observa gran cantidad de células superficiales, de forma poliédrica y de núcleo picnótico que en ocasiones no se llega a ver, pudiéndose además encontrarse células superficiales anucleares; en el metaestro se observa escasa cantidad de leucocitos, células intermedias y reducida cantidad de células superficiales; y en el diestro se nota la mayor cantidad de leucocitos pero escasa cantidad de células intermedias en proporción a los leucocitos (Kühnel y Mendoza, 1992 ).

El cuerpo lúteo (CL) se caracteriza por la presencia de células de la granulosa luteínicas y de las células tecales luteínicas. Las primeras, llamadas también células luteínicas grandes, son poligonales, de citoplasma abundante y pálido, con núcleo grande y vesicular, actividad mitótica baja, y ocupan la mayor parte del CL, y las segundas, llamadas también células luteínicas pequeñas, son más pequeñas que las anteriores, con citoplasma oscuro, núcleo pequeño y basófilo, y se encuentran mayormente en las zonas trabeculares y periféricas del CL (Eroschenko y di Fiore, 2008).

Shi et al. (2000) documentaron las concentraciones hormonales en el ciclo estral del cuy, encontrando que la concentración de FSH alcanza los niveles más bajos y los de estradiol los niveles más altos en el día de la ovulación (día 0). Después de la ovulación, los niveles de FSH se incrementan y los de estradiol disminuyen. La concentración de progesterona es baja en los días 16 y 0 , y alta entre los días 4 a 12. Asimismo, la LH es alta en el día 0 , disminuyendo luego de la ovulación y aumentando nuevamente en el día 12 (Blatchley et al., 1976). 
En el Perú, se han desarrollado razas y líneas de cuyes que permitan mejorar los índices productivos de su crianza. Entre las razas más promisorias se encuentran Perú y Andina (desarrolladas por el Instituto Nacional de Innovación Agraria - INIA). La raza Perú se caracteriza por una rápida ganancia de peso en una menor unidad de tiempo y con un tamaño de camada de 2.6 crías, en tanto que la raza Andina destaca por un mayor tamaño de camada (3.6 crías por camada) (INIA, 2013).

La existencia de varias razas de cuyes justifica la investigación sobre la fisiología reproductiva básica a fin de explicar las diferencias en el comportamiento reproductivo y tener fundamento científico que apoye en la toma de decisiones técnicas en los sistemas de crianza. El presente tuvo por objetivo determinar el número promedio de folículos que llegan a ovular en cuyes de las razas Andina y Perú, con el fin de saber si la diferencia en el tamaño de camada entre estas razas se podría deber, en parte, al número de ovulaciones.

\section{Materiales y Métodos}

\section{Localización y Animales}

El estudio se realizó en el Instituto Nacional de Investigación Agraria (INIA), ubicado en el distrito de La Molina, Lima, entre los meses de noviembre de 2008 y marzo de 2009. El procesamiento de las muestras histológicas se realizó en el Laboratorio de Patología de la Facultad de Veterinaria y Zootecnia, Universidad Peruana Cayetano Heredia, Lima.

El tamaño mínimo muestral fue calculado en 15 hembras por raza. Los animales eran cuyes hembras púberes de 10 semanas de edad de las razas Andina y Perú. Los cuyes se distribuyeron en grupos de 7 y 8 individuos en pozas de 1.0 × $1.5 \times 0.8 \mathrm{~m}$ y recibieron alimento de alta densidad nutricional (18\% de proteína y $2.8 \mathrm{Mcal} / \mathrm{kg}$ de alimento) en pellets, ad libitum, así como maíz chala (Zea mays) fresca a razón de $10 \%$ del peso vivo del animal y agua ad libitum. No hubo presencia de machos en las pozas durante todo el estudio.

\section{Diseño Experimental}

\section{Tamaño de la muestra}

Se obtuvo mediante la fórmula de diferencia de medias, donde se consideró una media de 3.24 ovulaciones por ciclo (Vigil, 1971) y una media de 4.71 ovulaciones por ciclo para los animales de la raza más prolífica (hipótesis del estudio). Asimismo, se consideró un nivel de confianza del 95\%, una potencia de la prueba del $80 \%$ y desviación estándar de 1.08 (obtenida a partir del conteo de CL de 10 hembras). El tamaño de muestra mínimo calculado fue de 15 hembras por raza.

\section{Frotices vaginales}

Las muestras de mucosa vaginal se obtuvieron con hisopos descartables estériles de 6" que fueron introducidos hasta el tercio medio de la vagina. Se hicieron frotices en láminas portaobjetos y se colorearon con tinción citológica rápida $\mathrm{T} 15 \circledR$. El frotis fue observado al microscopio óptico a 400x.

\section{Determinación del día de la ovulación}

Los animales se revisaron en forma diaria hasta observar la ausencia de membrana vaginal (Sadeu et al., 2007). A partir de allí, se realizaron frotices vaginales diarios que permitieron determinar el día de la ovulación (día 0) (Shi et al., 2000), mediante la presencia del máximo de cornificación de las células superficiales y antes de la aparición de leucocitos. 


\section{Detección del diestro}

El día 8 del ciclo estral es parte del diestro y se tiene la presencia de CL (Westfahl, 1988). En la citología vaginal solo deben aparecer células intermedias con gran cantidad de leucocitos (Kühnel y Mendoza, 1992).

\section{Evaluación histológica del ovario}

Los ovarios fueron fijados con formol buferado al $10 \%$ por $48 \mathrm{~h}$. Se les hizo cortes longitudinales seriados de $5 \mu \mathrm{m}$ de espesor y se tiñeron con Hematoxilina y Eosina (Luna, 1968). Se observaron 10 cortes histológicos por ovario en microscopio óptico a 100 y $400 \mathrm{x}$ para el conteo de los CL (Bland, 1966). La confirmación de la funcionalidad del CL se basó en la presencia de mitosis en las células de la granulosa luteínicas (Azmi et al., 1984).

\section{Análisis de Datos}

Cada CL funcional hallado en los ovarios fue interpretado como folículo maduro que ovuló en el estro que precedió a la toma de muestra. Se utilizó el diagrama de CajaBigotes para evaluar la distribución del número de CL totales.

La normalidad de la variable número de CL fue probada mediante la prueba de Kolmogorov Smirnov de una muestra y la diferencia entre el número de CL entre las razas se determinó mediante la prueba de $\mathrm{T}$ de Student, usando el programa SPSS 13.0.

\section{Resultados}

Uno de los cuyes de la raza Andina presentó un solo cuerpo lúteo; resultado que fue considerado atípico y, por lo tanto, excluido del estudio. La raza Andina presentó un mayor número de CL que la raza Perú $(\mathrm{p}<0.05)$ en el día 8 del ciclo estrual, en tanto que el número de CL del ovario derecho e izquierdo fue estadísticamente similar (Cuadro 1). No obstante, se encontró una mayor presencia de CL en el ovario derecho de cuyes de la raza Andina que en aquellos de la raza Perú (Cuadro 2).

Los hallazgos citológicos obtenidos en las cuatro fases del ciclo estrual se pueden observar en la Fig. 1. Asimismo, las características histológicas del CL se muestran en la Fig. 2.

El cuerpo lúteo se encontró delimitado por tejido conectivo periférico conformado por fibroblastos y vasos sanguíneos (Fig. 2a). Se observaron células de la granulosa luteínicas ocupando la mayor parte del CL. Estas células eran ovoides, de citoplasma amplio con una o varias vacuolas, y de núcleo vesiculoso (Fig. 2d). Las células tecales luteínicas son de núcleo basófilo muy pequeño y citoplasma reducido (Fig. 2b). Los capilares sanguíneos se ubican tanto en la periferie como en el centro del CL (Fig. 2d). Los capilares del centro tienen el lumen más reducido debido a la acción expansiva de las células de la granulosa luteínica, que presentan una actividad mitótica baja (Fig. 2c). Todos los CL evaluados cumplían con el requisito de actividad mitótica.

\section{Discusión}

Los hallazgos en los frotices vaginales coinciden con lo reportado por Kühnel y Mendoza (1992).

En especies de ovulación múltiple se pueden esperar diferencias entre razas en el número de ovulaciones por ciclo estrual. Así por ejemplo, en el ovino existe diferencias importantes en la tasa de ovulación entre la Merino (1.2 óvulos) y la Finnish Landrace (3 óvulos) (Hafez, 2003). En el presente estudio, se encontró una mayor tasa de ovulación en la raza Andina, la cual se conoce como raza prolífica por el mayor tamaño de camada (INIA, 2005). 
Cuadro 1. Número de cuerpos lúteos en cuyes (Cavia porcellus) en el día 8 del ciclo estrual

\begin{tabular}{lllcccc}
\hline \multirow{2}{*}{ Variable } & & \multirow{2}{*}{$\mathrm{n}$} & Media & \multirow{2}{*}{$\begin{array}{c}\text { Desviación } \\
\text { estándar }\end{array}$} & \multicolumn{2}{c}{ Rango } \\
\cline { 6 - 8 } Raza & Andina & 14 & $3.6^{\mathrm{a}}$ & 0.9 & 1 & 5 \\
& Perú & 15 & $2.8^{\mathrm{b}}$ & 0.9 & 1 & 4 \\
\multirow{2}{*}{ Ovario } & Derecho & 29 & $1.6^{\mathrm{a}}$ & 0.9 & 0 & 4 \\
& Izquierdo & 29 & $1.6^{\mathrm{a}}$ & 1.1 & 0 & 4 \\
\hline
\end{tabular}

${ }^{a, b}$ Superíndices diferentes dentro de cada variable indican diferencias estadísticas $(p<0.05)$

Cuadro 2. Número de cuerpos lúteos en cuyes (Cavia porcellus) en el día 8 del ciclo estrual en las razas Andina y Perú se gún el ovario donde se localizan

\begin{tabular}{lcccccc}
\hline \multirow{2}{*}{ Raza } & \multicolumn{3}{c}{ Ovario derecho } & \multicolumn{3}{c}{ Ovario izquierdo } \\
\cline { 2 - 7 } & $\mathrm{n}$ & Media & $\mathrm{DE}$ & $\mathrm{n}$ & Media & $\mathrm{DE}$ \\
\hline Andina & 14 & $1.9^{\mathrm{a}}$ & 0.8 & 14 & $1.6^{\mathrm{a}}$ & 1.2 \\
Perú & 15 & $1.2^{\mathrm{b}}$ & 0.5 & 15 & $1.6^{\mathrm{a}}$ & 1.3 \\
\hline
\end{tabular}

${ }^{a, b}$ Superíndices diferentes dentro de columnas indican diferencias estadísticas $(p<0.05)$

El ovario derecho tiende a ser más activo en término de ovulaciones que el ovario izquierdo. Esto se puede observar en la vaca (Cushman et al., 2005), oveja (Hafez, 2003) y yegua (Preildkalns, 1993), donde alrededor del 55-65\% de las ovulaciones ocurren en el ovario derecho. No obstante, la actividad ovárica en el cuy presentó una tasa de ovulación similar entre ambos ovarios.

Un estudio anterior demostró que el cuy produce $3.4 \pm 0.4$ óvulos por ciclo estral (Noonan, 1994). Por otro lado, en el presente estudio se encontró $3.6 \pm 0.9$ y $2.8 \pm 0.9$ ovulaciones para las razas Andina y Perú, respectivamente. La diferencia entre razas podría deberse a la orientación que ha tenido el programa de mejoramiento genético, donde los animales de la raza Perú fueron seleccionados por ganancia de peso y precocidad, en tanto que los de la raza Andina fueron seleccionados por prolificidad (INIA, 2013). Así mismo, el INIA reporta tamaños de camada al primer parto de 2.9 y 2.2 crías para estas razas (INIA 2013), lo cual permite plantear que existiría un alto índice de óvulos no fecundados y/o mortalidad embrionaria en esta especie.

El tamaño de camada es el resultado del número de ovulaciones, de la tasa de óvulos no fecundados y de la tasa de mortalidad embrionaria. En el caso del conejo, razas como California y Neozelanda tienen ocho 


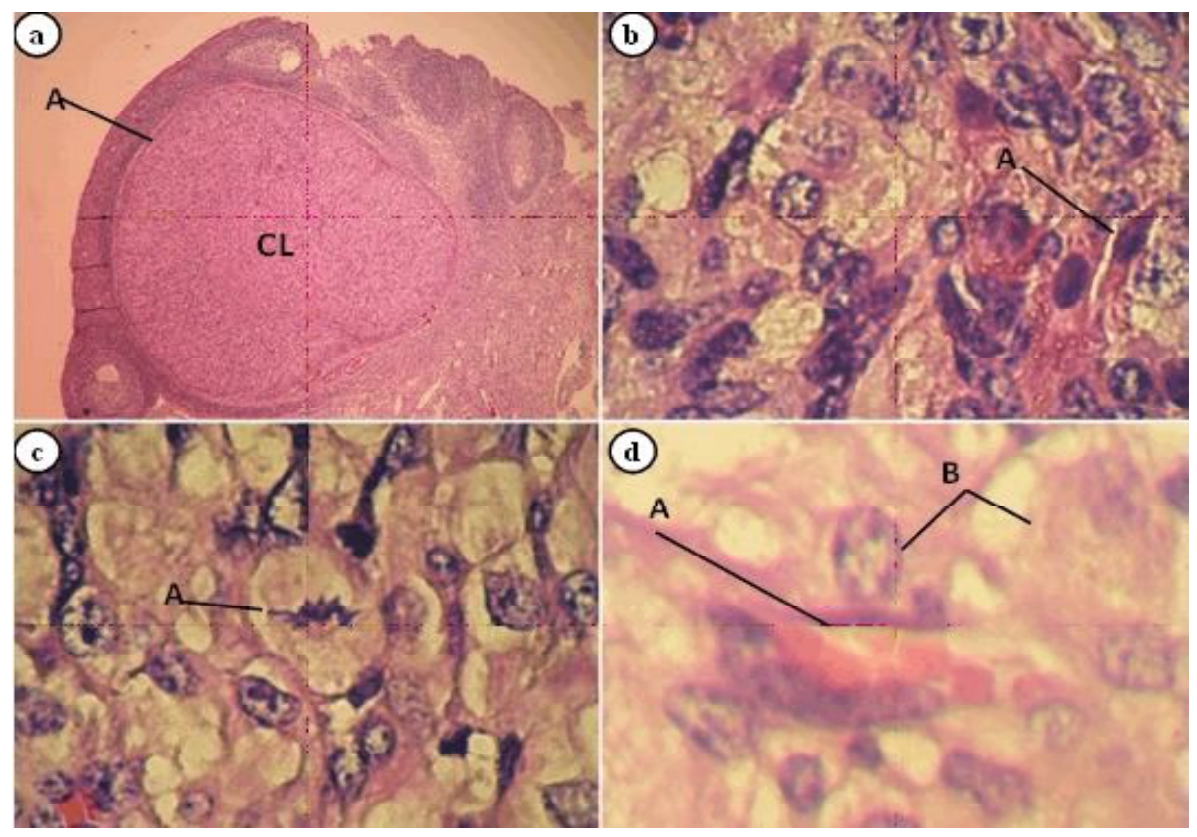

Figura 1. Citología vaginal del cuy. (a) Diestro: Gran cantidad de leucocitos en relación a las células intermedias, 400X, HE; (b) Proestro: Gran cantidad células intermedias y presencia de mucus, 100X, HE; (c) Estro: Predominio exclusivo de células superficiales. 100X, HE; (d) Metaestro: Regular número de leucocitos y mayor cantidad de células intermedias en relación a las superficiales, 400X, HE

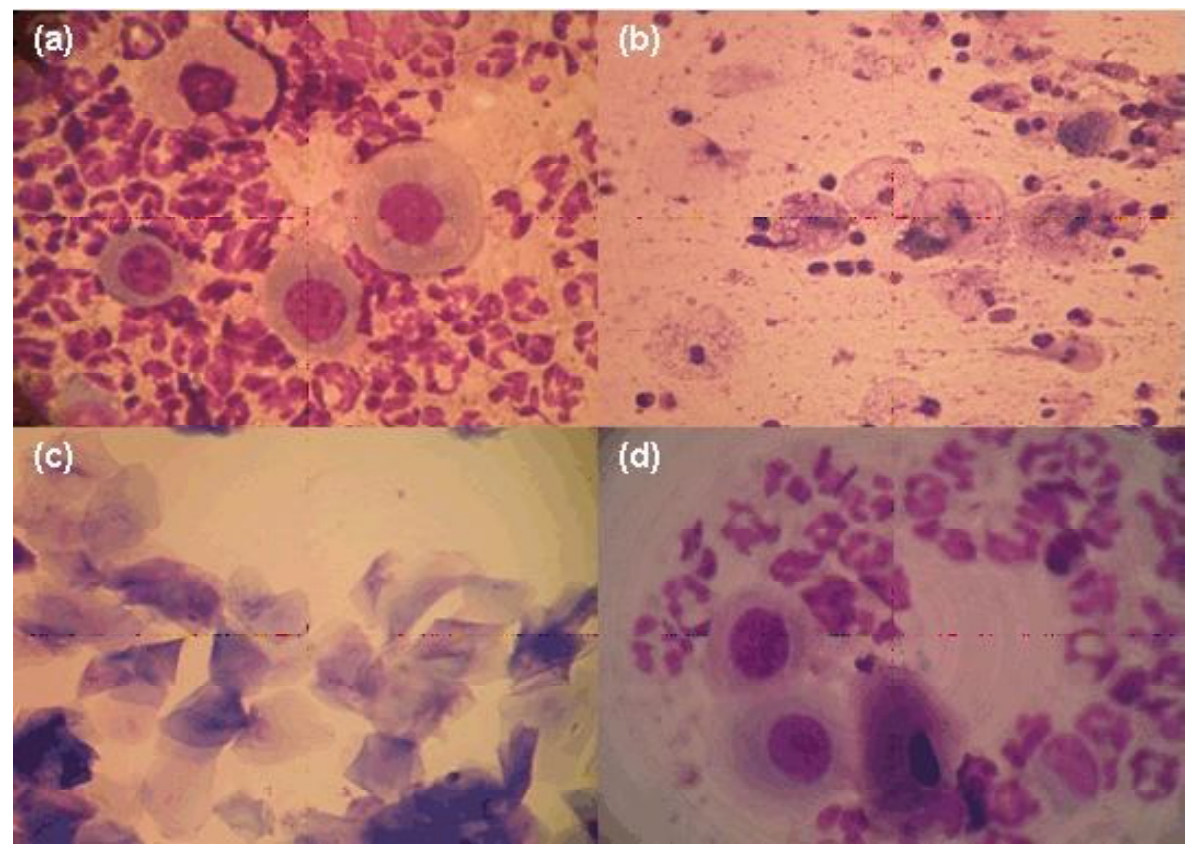

Figura 2. Corte transversal del cuerpo lúteo del cuy. (a) A: Tejido conectivo, CL: Cuerpo lúteo, 100X, HE; (b) A: célula tecal luteínica, 400X, HE; (c) A: Célula de la granulosa luteínica en mitosis, 400X, HE; (d) A: Vaso sanguíneo, B: Células de la granulosa luteínicas, 400X, HE 
gazapos al parto, pero la primera tiene $11.6 \mathrm{y}$ la segunda tiene 9.6 ovulaciones por ciclo estrual. La explicación se debe en parte a que la raza California tiene entre 30 a $37 \%$ de óvulos no fecundados (Walker et al., 1997) frente al 5-12\% de la raza Neozelanda, además de una menor pérdida embrionaria (Alvariño, 1993). Estas diferencias reproductivas entre razas de conejos, sugieren que puede haber diferencias en el porcentaje de óvulos no fecundados y de mortalidad embrionaria entre razas de cuyes.

\section{Conclusiones}

- La media de ovulaciones por ciclo estrual fue de 3.6 y 2.8 para las razas Andina y Perú, respectivamente.

- No hubo diferencias estadísticas en el número de ovulaciones entre el ovario izquierdo y derecho.

- La diferencia en el tamaño de camada entre las dos razas se podría deber, en parte, a que la raza Andina tiene mayor número de ovulaciones por ciclo estral que la raza Perú.

\section{Literatura Citada}

1. [INIA] Instituto Nacional de Innovación Agraria. 2013. Cuyes. [Internet], [15 septiembre 2013]. Disponible en: http://www.minag.gob.pe/portal/sectoragrario/pecuaria/situacion-de-las-actividades-de-crianza-y-produccion/cuyes

2. Alvariño M, Ubilla E. 1993. Fisiología de la reproducción en la hembra. En: Alvariño M (ed). Control de la reproducción en el conejo. Madrid: Mundi-Prensa. p 33-50.

3. Azmi TI, O'Shea JD, Bruce NW, Rodgers RJ. 1984. Morphometry of the functional and regressing corpus luteum of the guinea pig. Anat Rec 210: 33-40.

4. Bland KP, Donovan BT. 1966. Uterine distension and the function of the corpora lutea in the guinea-pig. J Physiol 186:503-515.
5. Chauca L. 1997. Producción de cuyes (Cavia porcellus). Rome: Food and Agriculture Organization of the United Nations. 77 p.

6. Cushman RA, Allan MF, Snowder GD, Thallman RM, Echternkamp SE. 2005. Evaluation of ovulation rate and ovarian phenotype in puberal heifers from a cattle population selected for increased ovulation rate. J Anim Sci 83: 1839-1844.

7. Eroschenko V, di Fiore M. 2008. DiFiore's atlas of histology with functional correlations. $11^{\circ}$ ed. Philadelphia: Lippincott Williams \& Wilkins. 523 p.

8. Hafez. 2003. Ovejas y cabras. En: Hafez ESE, Hafez B (eds). Reproducción e inseminación artificial en animales. $7^{\circ}$ ed. México: McGraw Hill. p 177-178.

9. Kühnel W, Mendoza A. 1992. Scanning electron microscope investigations on the vaginal epithelium of the guinea pig during the estrous cycle. Arch Histol Cytol 55: 205-210.

10. Luna F, Cortés M, Flores $M$, Hernández B, Trujillo A, Domínguez $\boldsymbol{R}$. 2003. The effects of superior ovarian nerve sectioning on ovulation in the guinea pig. Reprod Biol Endocrinol 1: 61.

11. Noonan D. 1994. The guinea pig ( $\mathrm{Ca}$ via porcellus). ANZCCART News 7(3): 1-7.

12. Preildkalns J. 1993. Sistema reproductor femenino. En: Dellman HD (ed). Histología veterinaria. $2^{\mathrm{a}}$ ed. Zaragoza: Acribia. p 267-290.

13. Sadeu JC, Adriaens I, Cortvrindt R, Smitz J. 2007. Study of folliculogenesis in vivo in guinea pig. Theriogenology 68: 1228-1239.

14. Shi F, Watanabe G, Trewin A, Hutz R, Taya K. 2000. Localization of ovarian inhibin/activin subunits in follicular dominance during the estrous cycle of guinea pigs. Zool Sci 17: 1311- 1320.

15. Vigil DV. 1971. Caracterización del ciclo estral en cobayos hembras vírgenes (Cavia porcellus). Tesis de Ingeniero Zootecnista. Lima: Universidad Agraria La Molina. 91 p. 
16. Walker D, De la Peña R, Rowley J, Somenov K, González F, Gutiérrez N, Fábregas E. 1997. Contribución genética a la mortalidad embrionaria en 2 poblaciones de roedores no consanguíneos. Rev Cubana Invest Biomed 16: 45-49.
17. Westfahl PK, Vekasy M. 1988. Changes in serum and ovarian steroids during reproductive development in the female guinea pig. Biol Reprod 39: 10861092. 